\title{
Digital Image Forgery Detection Based on Characteristics of Background Noise
}

\author{
Jiming Zheng ${ }^{1,2, a}$, Guoyu Zhou ${ }^{1}$, Jinling Geng ${ }^{2}$, Qinghua Zhang ${ }^{2}$ \\ ${ }^{1}$ School of Computer Science and Technology, Chongqing University of Posts and \\ Telecommunications, Chongqing, 400065, China \\ ${ }^{2}$ School of Science, Chongqing University of Posts and Telecommunications, Chongqing, 400065, \\ China \\ aemail: zhengjm@cqupt.edu.cn
}

Keywords: Image forensics; Background noise; Wavelet transform ; Skewness

\begin{abstract}
In this paper, we proposed a method to detect splicing forgery by using wavelet transform and background noise estimation in digital image. To achieve this target, we divide image into some sub-blocks and use wavelet transform to extract the low-frequency sub-band coefficients of each ones. Then, the noise standard deviation in each bands can be estimated with the statistical properties of skewness. In the end, the morphological marker is used to tamper with the region. Comparisons with existing methods by experiments showed that our proposed method is effective in estimating the noise standard deviation, and has high accuracy in detecting and localizing forgery parts especially in local smooth regions.
\end{abstract}

\section{Introduction}

Image splicing forgery is a common type of image tampering, which copies a region from the source image, and then pastes it into the target image to conceal the target image information. In fact, the complex splicing forgery usually contains some post-processing operations to make the tampered image more real. These operations include blurring, shading, JPEG compression, etc. It will make forgery detection and localization more difficult. In recent years, some detection techniques for splicing forgery detection have achieved good results [1, 2].

There is a part of background noise in digital image which comes from image generation. In the case of untampered images, the noise standard deviation in different areas is substantially same. If images with different noise levels are spliced together, the noise characteristics between image forgery area and the other areas are different. Many conventional forgery detection methods are exposing splicing by estimating background noise. Popescu et al[3] treat the image as additive noise model, and use the high order statistics to estimate the noise variance. However, their method needs to know the kurtosis of the original signal. In order to estimate noise standard deviation of each non-overlapping block, the wavelet transform is used in [4]. Feiyan Lu et al[5] directly consider the detected image as the original image and add noise twice, so they avoid the problem in method [3] that the original signal are unknown. The method of using statistical features to locate the tampered area is introduced in $[6,7]$. They utilize statistical relationship between the noise standard deviation and kurtosis or skewness. However, the proposed method has a higher false positive in smooth regions. Due to the image after wavelet transform, the noise in the wavelet domain still has the independent and identically distributed characteristics. In this paper, the image to be detected is decomposed by wavelet transformation, and we use the skewness characteristic of low-frequency sub-band coefficients to estimate the noise standard deviation. Our method is more effective to avoid the interferences of image details when estimating the noise standard deviation, thereby detecting forgery splicing area. In order to improve detection accuracy, we use labeled connected component method to reduce false information, and utilize morphological operations to show the forgery area clearly. 


\section{Improved Method of Estimating the Background Noise}

\section{- Wavelet transform}

When an image is decomposed by wavelet transform, we can get a series of sub images with different resolutions, and the corresponding frequency of the sub images of different resolutions is not the same. Figure 1 shows a tower structure formed by the multi-level wavelet decomposition. HL2, HH1 and HL1 has a higher resolution and save more image information compared with sub image LH2, HH2, LH1.

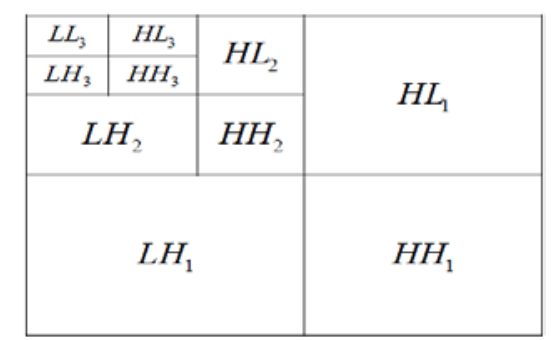

Fig.1. The tower structure of multi-level wavelet decomposition

Haar function is one of the most earliest used in wavelet analysis with a compact support orthogonal wavelet function. In order to reduce the interference of image details on noise detection, for an image $I$ of $M \times N$ pixels, the 1-level 2-D Haar wavelet transform is adopted. We only retain total of $K$ coefficients of low-frequency sub-band $L L_{1}$ in each sub-block, and utilize statistical properties of skewness in $L L_{1}$ coefficients to estimate noise variance, where $K$ is $\frac{M}{2} \times \frac{N}{2}$.

\section{- The statistical properties of skewness for background noise}

Assuming the model in the time and frequency domain of the additive noise image $I$ is $y=x+z$, where $y, x, z$ are obtained respectively, when additive noise image $Y$, original image $X$, noise $Z$ are transformed into the time and frequency domain from the pixel domain. $z$ is white Gaussian noise whose mean is zero and variance is $\sigma^{2}(z)$. We estimate $\sigma^{2}(z)$ by using the statistical characteristics of $x$ and $y$ as follows.

Let the mean value and variance of $x$ be $E(x)$ and $\sigma^{2}(x)$ respectively. Then we derive the skewness of $x$ as follows:

$$
\omega(x)=\frac{\mu_{3}(x)}{\sigma^{3}(x)}
$$

where $\sigma^{3}(x)=\left\{\sigma^{2}(x)\right\}^{\frac{3}{2}}=\left\{E\left\{(x-E(x))^{2}\right\}\right\}^{\frac{3}{2}}, \mu_{3}(x)=E\left\{(x-E(x))^{3}\right\}$.

Since $z$ is a white noise, $\mu_{3}(z)=E\left\{(z-E(z))^{3}\right\}=E\left(z^{3}\right)$, according to the parity of the integral, we have $\mu_{3}(z)=0$. Hence, skewness of $z$ is

$$
\omega(z)=\frac{\mu_{3}(z)}{\sigma^{3}(z)}=0
$$

Further, because $x$ and $z$ are independent to each other, the variance and skewness of $y$ can be calculated as follows:

$$
\begin{aligned}
& \sigma^{2}(y)=\sigma^{2}(x)+\sigma^{2}(z) \\
& \omega(y)=\frac{\mu_{3}(y)}{\sigma^{3}(y)}=\frac{\mu_{3}(x)+\mu_{3}(z)}{\sigma^{3}(y)}=\frac{\omega(x) \sigma^{3}(x)}{\sigma^{3}(y)}=\omega(x)\left(\frac{\sigma^{2}(y)-\sigma^{2}(z)}{\sigma^{2}(y)}\right)^{\frac{3}{2}}
\end{aligned}
$$

Therefore, we get the relationship between the values of skewness and noise deviation. We can estimate skewness and the noise deviation of the image to be detected. It is an objective function of minimizing as follows[6,7]: 


$$
\left[\hat{\omega}(x), \hat{\sigma}^{2}(z)\right]=\min _{\omega(x), \sigma^{2}(z)} \sum_{k=1}^{K}\left[\omega\left(y_{k}\right)-\omega(x)\left(\frac{\sigma^{2}\left(y_{k}\right)-\sigma^{2}(z)}{\sigma^{2}\left(y_{k}\right)}\right)^{\frac{3}{2}}\right]^{2}
$$

where $y_{k}$ is represented each sub-band, $k \in\{1,2, \ldots, K\}$. Then Eq. (4) can be changed as follows:

$$
\omega^{\frac{2}{3}}(y)=\omega^{\frac{2}{3}}(x)-\omega^{\frac{2}{3}}(x) \frac{\sigma^{2}(z)}{\sigma^{2}(y)}
$$

So we have

$$
\left[\hat{\omega}^{\frac{2}{3}}(x), \hat{\sigma}^{2}(z)\right]=\min _{\omega(x), \sigma^{2}(z)} \sum_{k=1}^{K}\left[\omega^{\frac{2}{3}}\left(y_{k}\right)-\omega^{\frac{2}{3}}(x)+\omega^{\frac{2}{3}}(x) \frac{\sigma^{2}(z)}{\sigma^{2}\left(y_{k}\right)}\right]^{2}
$$

Then, let the objective function be as:

$$
L\left(\omega^{\frac{2}{3}}(x), \sigma^{2}(z)\right)=\sum_{k=1}^{K}\left[\omega^{\frac{2}{3}}\left(y_{k}\right)-\omega^{\frac{2}{3}}(x)+\omega^{\frac{2}{3}}(x) \frac{\sigma^{2}(z)}{\sigma^{2}\left(y_{k}\right)}\right]^{2}
$$

using partial derivative principle obtains the optimal solution as follows:

$$
\begin{gathered}
\hat{\omega}^{\frac{2}{3}}(x)=\frac{<\omega^{\frac{2}{3}}\left(y_{k}\right)>_{K}<\frac{1}{\left[\sigma^{2}\left(y_{k}\right)\right]^{2}}>_{K}-<\frac{\omega^{\frac{2}{3}}\left(y_{k}\right)}{\sigma^{2}\left(y_{k}\right)}>_{K}<\frac{1}{\sigma^{2}\left(y_{k}\right)}>_{K}}{<\frac{1}{\left[\sigma^{2}\left(y_{k}\right)\right]^{2}}>_{K}-<\frac{1}{\sigma^{2}\left(y_{k}\right)}>_{K}^{2}} \\
\hat{\sigma}^{2}(z)=\frac{1}{<\frac{1}{\left[\sigma^{2}\left(y_{k}\right)\right]^{2}}>_{K}}-\frac{1}{\omega^{\frac{2}{3}}(x)} \frac{<\omega^{\frac{2}{3}}\left(y_{k}\right)>_{K}}{\frac{1}{\sigma^{2}\left(y_{k}\right)}>_{K}}
\end{gathered}
$$

where $\langle\cdot\rangle_{K}$ denotes the mean of $K$ sub-band coefficients. Combined Eq.(9) and (10), we can estimate the standard deviation of noise $\hat{\sigma}(z)=\sqrt{\hat{\sigma}^{2}(z)}$.

\section{Image tamper detection algorithm flow}

According to the above analysis, the algorithm divide image into some sub-blocks, and then use wavelet transform to extract the low-frequency sub-band coefficients of each ones. Next, the noise standard deviation in each bands can be estimated with the statistical properties of skewness, and morphological processing is performed to show the tampered region. The flow of the detection algorithm is as follows:

(1) Treate the image as additive noisy image, and divide the image into blocks.

(2) Wavelet transform is performed on each block and extract the low-frequency coefficients of the corresponding frequency, then get $K$ coefficients sub-bands.

(3) For each sub-band, compute skewness $\omega\left(y_{k}\right)$ and variance $\sigma^{2}\left(y_{k}\right)$, and then calculate the noise standard deviation of all the sub-bands with Eq. (9) and Eq. (10), where $k \in\{1,2, \ldots, K\}$.

(4) Label the connected componets and use morphological operations to clearly show the forgery area.

There are overlapped block and non-overlapping block in image blocking methods. According to the experimental results, the algorithm will use overlapping block as shown in Figure 2. It is half the length of the block from left to right, top to bottom in the order to traverse the entire image. For an image $I$ of $M \times N$ pixels and the sub-block size is $a \times a$, a total of $\left\lfloor\frac{M}{a / 2}-1\right\rfloor \times\left\lfloor\frac{N}{a / 2}-1\right\rfloor$ sub-blocks can be divided. According to the experimental results in next, the sub-block size should be moderate, and when the algorithm uses $16 \times 16$ overlapping blocks, the detection effect is more accurate. 

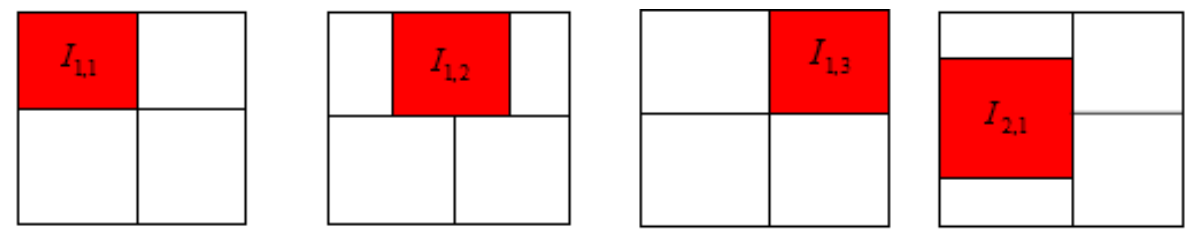

Fig.2. Overlapping block

In morphological processing, most of the tampered regions are contiguous blocks, and misjudgment regions are usually isolated and dispersed points. In order to reduce the error, labeling the connected componets[8] (shown in Figure 3) is used to make detection more accurate. From Figure 3 we can see that threshold $T=3$ is more accurate than $T=2$, but $T=4$ will cause more time or more error detections than $T=3$, so we set $T=3$.

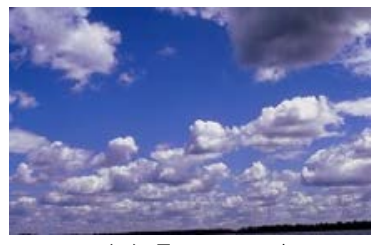

(a) Image $\mathrm{A}$

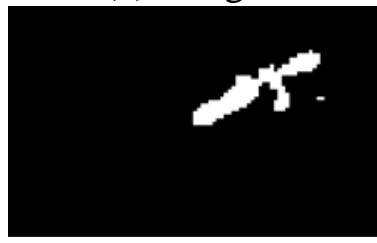

(e) $T=1$

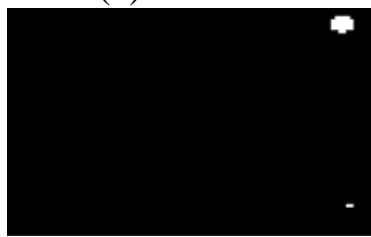

(i) $T=1$

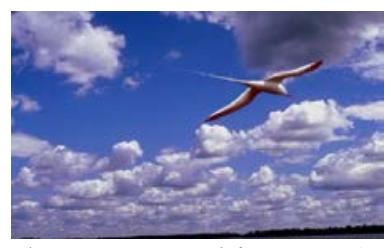

(b)Tampered image A

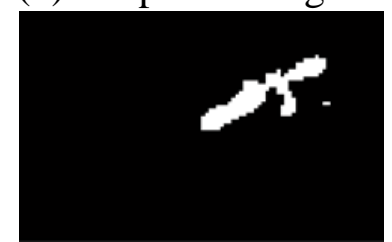

(f) $T=2$

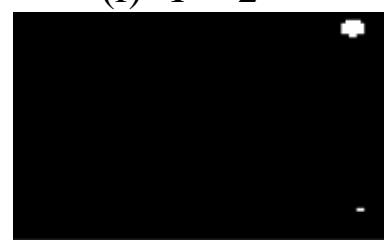

(j) $T=2$

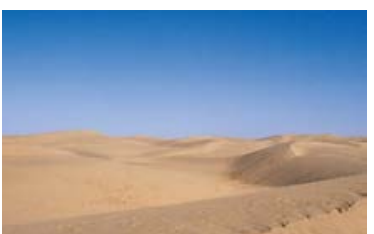

(c)Image B

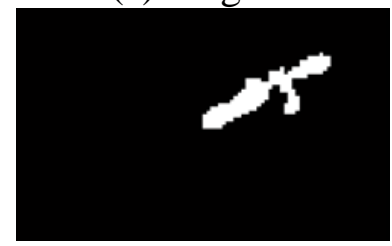

(g) $T=3$

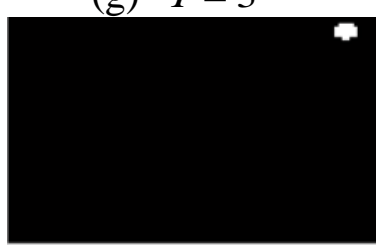

(k) $T=3$

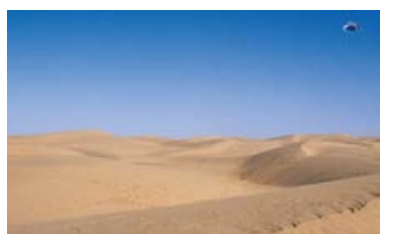

(d)Tampered image B

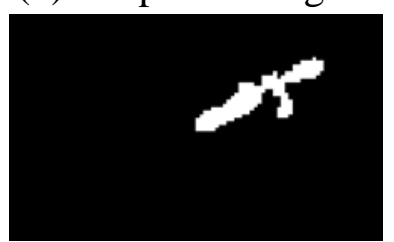

(h) $T=4$

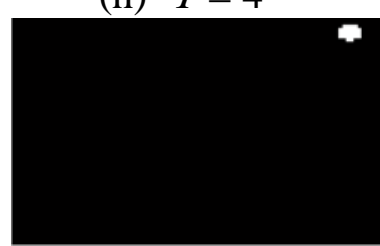

(l) $T=4$

Fig.3. Label the size of connected components to eliminate errors. The first row are two original images and two tamperd images, the second row is the result of tamperd image A, the third row is the result of tamperd image $\mathrm{B}$.

\section{Experimental Results and Comparisons}

\section{- Noise standard deviation estimation}

In order to evaluate the accuracy of the algorithm estimates noise standard deviation in local smooth regions, we select 50 authentic images from CASIA v2.0[9] dataset. The selected images include natural scene, animals and people, etc. And the local smooth regions just like sky, sea, lake and so on are shown in Figure 4. In the experiment, we add different noise standard deviation of Gaussian white noise, then the image is blocked and transformed by wavelet, finally we combine Eq.(9) and (10) to estimate the standard deviation of noise. For each image with different standard deviation, we compute the average and compare with the traditional wavelet method [4] and the method in [7], the results are shown in Table 1. We also add a series of different shapes white Gaussian noise with $20 \mathrm{~dB}$ standard deviation, and use our method to show visualization results, then compared with method in [8], finally the results are shown in Figure 5.

Table 1 and Figure 5 show that our method in local noise estimation ability will be more accurate than method in [4] and [7]. These experimental results are both examined with $16 \times 16$ overlapping sub-blocks. According to Figure 5, the method in [7] has more error detections than ours. 

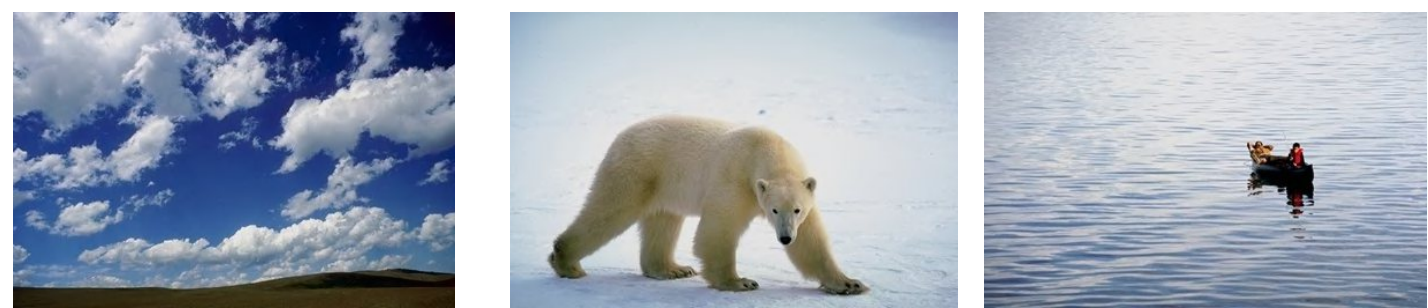

Fig.4. The example of authentic images from CASIA v2.0[9] dataset

Tab.1. Average of noise standard deviation comparison with different methods

\begin{tabular}{c|c|c|c|c|c}
\hline$\sigma$ & 0.5 & 1 & 5 & 10 & 20 \\
\hline \multirow{2}{*}{$\begin{array}{c}\text { Method in [4] } \\
\text { Method in [7] }\end{array}$} & 1.34 & 1.54 & 5.56 & 10.46 & 20.78 \\
\cline { 2 - 6 } Our method & 0.44 & 0.94 & 4.81 & 9.67 & 19.32 \\
\cline { 2 - 6 } & 0.49 & 0.97 & 4.86 & 9.70 & 19.65 \\
\hline
\end{tabular}
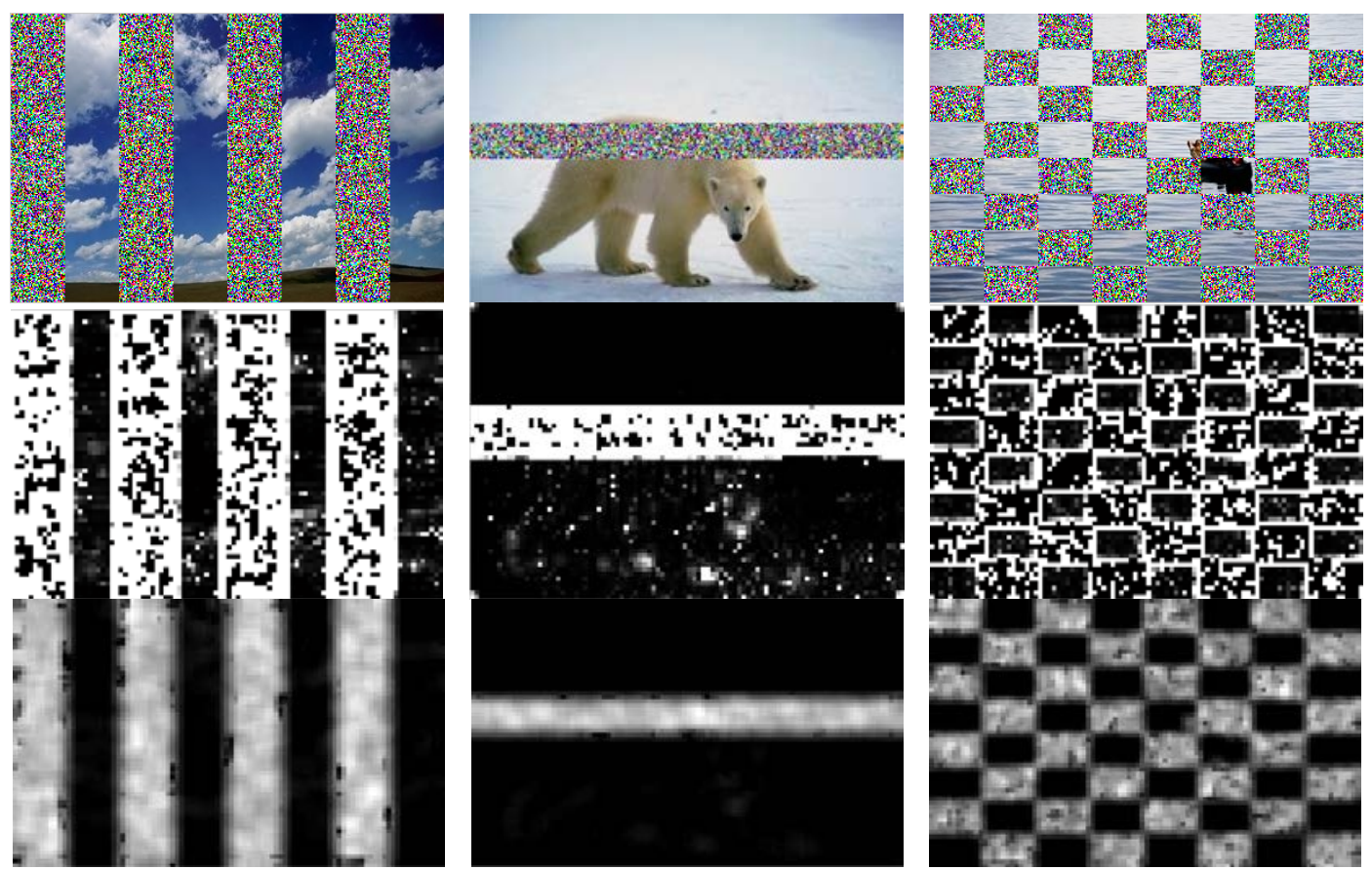

Fig.5. The comparison with [7] in local noise estimation, the first row is the noise-corrupted image, the second row is the method in [7], the last row is our method.

\section{- Tampered regions localization}

In this experiment, in order to evaluate the proposed algorithm how localizing tampered areas, we will use CASIA v2.0[9] dataset to evaluate our algorithm, and the image size is from $240 \times 160$ to $900 \times 600$ pixels. As we mentioned above, we should select the appropriate size of the sub-block according to the size of the image, and we choose an image from dataset [9] to carry out experiments to get the appropriate sub-block size. Figure 6 is the detection result of the different kinds of overlapping and non-overlapping sub-block, it shows that the image should be adopted in $16 \times 16$ overlapping sub-blocks method.

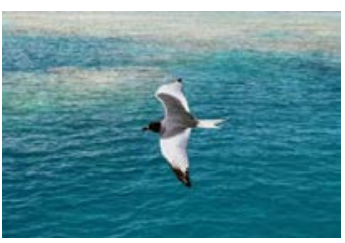

(a)

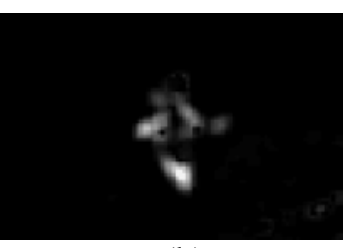

(b)

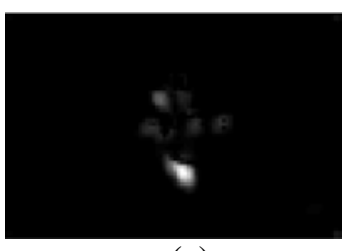

(c)

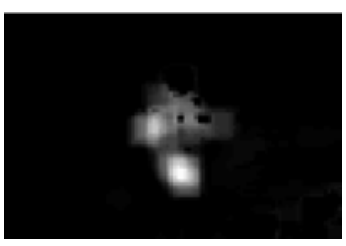

(d)

Fig.6. The detection result with different kinds of overlapping and non-overlapping sub-blocks, (a) is the tampered image, (b) is $16 \times 16$ overlapping sub-blocks, (c) is $16 \times 16$ non-overlapping sub-blocks, (d) is $32 \times 32$ overlapping sub-blocks. 
We make a comparison with algorithm presented in [7] based on CASIA v2.0 dataset. We selected 50 images to test the effectiveness of the algorithm. Figure 7 shows a few examples. In Figure 7, the white is the tampered area, the black is the original area, the first row is the original image, the second row is the tampered image, the third row is the result of the method in [7], the last row is our method. Especially, in the local smooth region, our method detects and locates the tampered area more accurately and effectively.

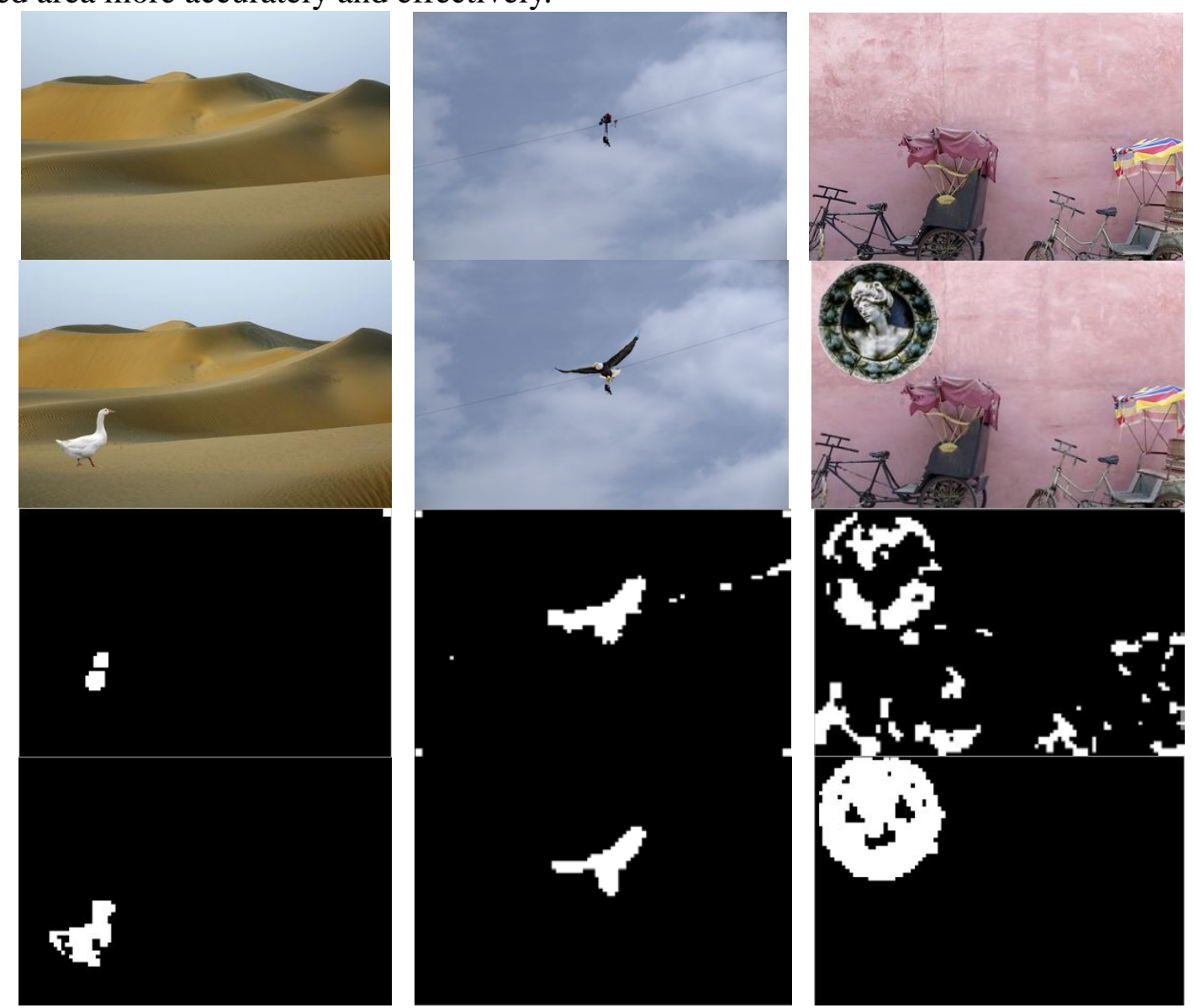

Fig.7. The detection result comparison with method in [7]

\section{Conclusion}

A splicing forgery detection approach based on wavelet transform and background noise estimation is proposed. We improved the method [7] by using wavelet transform to extract the low-frequency sub-band coefficients and labeling the size of connected components to eliminate errors. Experimental results show that the improved method can estimate the noise standard deviation accurately, and especially in the local smooth region, it can detect and locate the tampered area more accurately.

\section{Acknowledgement}

This paper was supported by Scientific and Technological Research Program of Chongqing Municipal Education Commission (Project No. KJ1400428) and the National Natural Science Foundation of China(Project No. 61472056).

\section{References}

[1] XIE Peng-cheng, LI Feng. Splicing Tampering Forensics of JPEG Images Based on the Resampling Detection[J]. Computing Technology and Automation, 2013, 32(1): 77-81. 
[2] Bahrami K, Kot A C, Li L, et al. Blurred Image Splicing Localization by Exposing Blur Type Inconsistency[J]. IEEE Transactions on Information Forensics and Security, 2015, 10(5): 999-1009.

[3] Popescu A C, Farid H. Statistical tools for digital forensics[C]. Information Hiding. 2004: 128-147.

[4] Mahdian B, Saic S. Using noise inconsistencies for blind image forensics[J]. Image and Vision Computing, 2009, 27(10): 1497-1503.

[5] LU Yan-fei, JU Ya-li, YU Yue. Image forgery detection using characteristics of background noise[J]. Signal Processing, 2012, 28(9): 1299-1307.

[6] Pan X, Zhang X, Lyu S. Exposing image forgery with blind noise estimation[C]. Proceedings of the thirteenth ACM multimedia workshop on Multimedia and security. 2011: 15-20.

[7] LIU Li-juan, Lin Xiao-zhu. Image forgery blind detection based on characteristics of background noise[J]. Computer Science, 2014, 41(B11): 136-138.

[8] Wu Shaobao, SHEN Dongsheng, Li Xiaowan. Blind detection of tampered image based on JPEG double quantization effect[J]. Computer Engineering and Applications, 2014, 50(20):202-206.

[9] National Lab of Pattern Recognition, Institute of Automation, Chinese Academy of Science. CASIA Tampered Image Detection Evaluation Database (CASIA TIDEv2.0) [EB/OL] source: http://forensics.idealtest.org. 